\title{
Reacceleration of electrons in supernova remnants
}

\author{
M. Pohl*, A. Wilhelm, I. Telezhinsky \\ Institute of Physics and Astronomy, University of Potsdam, Karl-Liebknecht-Strasse 24/25, \\ 14476 Potsdam, Germany \\ DESY, Platanenallee 6, 15738 Zeuthen, Germany
}

\begin{abstract}
The radio spectra of many shell-type supernova remnants show deviations from those expected on theoretical grounds. In this paper we determine the effect of stochastic reacceleration on the spectra of electrons in the $\mathrm{GeV}$ band and at lower energies, and we investigate whether reacceleration can explain the observed variation in radio spectral indices. We explicitely calculated the momentum diffusion coefficient for 3 types of turbulence expected downstream of the forward shock: fast-mode waves, small-scale non-resonant modes, and large-scale modes arising from turbulent dynamo activity. After noting that low-energy particles are efficiently coupled to the quasi-thermal plasma, a simplified cosmic-ray transport equation can be formulated and is numerically solved. Only fast-mode waves can provide momentum diffusion fast enough to significantly modify the spectra of particles. Using a synchrotron emissivity that accurately reflects a highly turbulent magnetic field, we calculated the radio spectral index and find that soft spectra with index $\alpha \leq-0.6$ can be maintained over more than 2 decades in radio frequency, even if the electrons experience reacceleration for only one acceleration time. A spectral hardening is possible but considerably more frequency-dependent. The spectral modification imposed by stochastic reacceleration downstream of the forward shock depends only weakly on the initial spectrum provided by, e.g., diffusive shock acceleration at the shock itself.
\end{abstract}

The 34th International Cosmic Ray Conference,

30 July- 6 August, 2015

The Hague, The Netherlands

\footnotetext{
* Speaker.
} 


\section{Introduction}

The synchrotron spectra observed from shell-type supernova remnants (SNR) are conventionally interpreted as being produced by electrons that have been accelerated at the forward shock and possibly the reverse shock through a process known as diffusive shock acceleration [1]. Whereas the electron spectrum at very high energies is shaped by energy losses and the structure of the cosmic-ray precursor, GeV-band electrons should not be affected by losses and boundary effects. Their spectrum should reflect the canonical solution $N(E) \propto E^{s}$ with $s=-2$ for strong shocks in monoatomic hydrogen gas. A slight softening of the spectra may arise from cosmic-ray feedback on the shock structure [2] and a proper motion of the cosmic-ray scattering centers, such that the compression ratio of the scattering centers is lower than that of the gas.

The GeV-scale spectrum of electrons is probed with measurements of their synchrotron emission in the radio band [3]. The values of the radio spectral index display a large scatter around a mean of $\alpha \approx-0.5\left(S_{v} \propto v^{\alpha}\right.$, with $\left.\alpha=(s+1) / 2\right)$, reaching in some cases $\alpha \approx-0.2$ or $\alpha \approx-0.8$, far beyond the level of systematic uncertainties in the measurements.

Here we re-examine the role of stochastic acceleration in SNR. It has been realized in recent years that non-resonant small-scale instabilities operating upstream in their non-linear phase impose substantial plasma turbulence that will foster second-order Fermi acceleration [4]. Secondary instabilities arise, for example by shock rippling, which lead to turbulent magnetic-field amplification downstream of the shock [5, 6], along with turbulent motions. Both on small and on large scales we therefore expect some second-order Fermi acceleration to operate behind the outer shocks of SNRs. Stochastic acceleration may thus act as a secondary re-acceleration process downstream of SNR shocks that slightly modifies the particle spectrum produced at the shock by diffusive shock acceleration.

In this paper we estimate the re-acceleration rate for three types of turbulence: fast-mode waves [7], Bell's non-resonant instability [8], and large-scale MHD turbulence arising from shock rippling through dynamo processes [9]. Having established the efficiency, energy dependence, and spatial decay scale of the momentum diffusion coefficient, we compute its effect on the spectrum of electrons between the forward shock and the contact discontinuity. We conclude with a discussion of the expected radio spectra of SNRs.

A full description of the methods and results is published elsewhere [10].

\section{The rate of diffusive reacceleration}

Before we discuss reacceleration rates in detail, it is important to recall that the downstream region of the forward shocks of young SNRs is not a low- $\beta$ environment. The flow is subsonic by definition, whereas the Alfvén speed increases only with the shock compression.

\subsection{Small-scale non-resonant modes}

Current-driven instabilities can lead to aperiodic small-scale turbulence [11, 8]. The saturation level of small-scale non-resonant modes is not well known. A significant backreaction is a reduction in streaming velocity and hence a diminishing of the streaming anisotropy [12]. Another non-linear side effect is turbulent motion [4]. 
The modes have a very low real frequency, at least in the linear stage, and so we can describe them with reasonable accuracy in the magnetostatic approximation. Using a second-order nonlinear theory of wave-particle interaction for a parametrized spectrum of strong magnetosonic slab turbulence we can estimate the momentum diffusion coefficient of relativistic particles with small Larmor radius, $r_{\mathrm{L}}$, $[13,14]$. If we equate the available time with the cascading timescale, $\tau_{d}$, at the largest scales [15], we can find the ratio of available time to the acceleration time, $p^{2} / D_{p}$, as

$$
\frac{\tau_{d} D_{p}}{p^{2}} \simeq \frac{\sqrt{\pi}}{3 s} \frac{\Gamma\left(\frac{s}{2}\right)}{\Gamma\left(\frac{s-1}{2}\right)} \frac{v_{\mathrm{A}}}{c}\left(\frac{r_{\mathrm{L}}}{r_{\mathrm{L}, \max }}\right)^{s-2}\left(\frac{\delta B}{B_{0}}\right)^{2} \Theta\left(r_{\mathrm{L}, \max }-r_{\mathrm{L}}\right) .
$$

It is typically small, unless $v_{A}$ is very large in the downstream region.

\subsection{Large-scale MHD turbulence}

The acceleration provided by moving magnetic-field structures is essentially a classical Fermi process. If scatterers at scale $k$ move with random velocity $v_{k}$ and the frequency of collision with these structures is $\omega_{k}$, then the momentum diffusion coefficient is determined as [16],

$$
D_{p} \approx p^{2} \int d \ln k \quad \omega_{k}\left(\frac{v_{k}}{c}\right)^{2} .
$$

Turbulence spectra are usually difficult to extract on account of the limited spectral range of MHD simulations. Simulations suggest that we may treat the turbulence structures as magnetic clouds of amplitude $B_{k}$ moving with random velocity $v_{k}$, where on the largest scales $v_{\mathrm{rms}} \simeq v_{\mathrm{sh}} / 20$ [17]. The scattering rate is then determined by the time needed to propagate between clouds, either ballistically or through diffusion with mean free path, $\lambda_{\mathrm{mfp}}$.

With Kolmogorov scaling the integral 2.2 yields

$$
D_{p} \approx \frac{2}{27 \pi c} p^{2} v_{\mathrm{rms}}^{2} \frac{\lambda_{\mathrm{mfp}} k_{\mathrm{min}}}{r_{\mathrm{L}}} .
$$

If the spatial diffusion follows Bohm scaling $\left(\lambda_{\mathrm{mfp}} \approx r_{\mathrm{L}}\right)$, then the acceleration time is independent of momentum. We then find for the acceleration time

$$
\tau_{\mathrm{acc}} \simeq \frac{p^{2}}{D_{p}} \approx\left(2.5 \cdot 10^{4} \mathrm{yr}\right)\left(\frac{0.03 c}{v_{\mathrm{sh}}}\right)^{2}\left(\frac{10^{-16} \mathrm{~cm}^{-1}}{k_{\min }}\right) \frac{r_{\mathrm{L}}}{\lambda_{\mathrm{mfp}}} .
$$

This is much longer than the evolutionary time scales of SNR, unless $k_{\min }$ is very small.

\subsection{Fast-mode waves}

The fastest waves in the downstream region should be fast-mode waves whose phase velocity is the sound speed, $v_{\mathrm{fm}} \simeq 1000 \mathrm{~km} / \mathrm{s}$. Particles can interact with fast-mode waves through transittime damping (TTD), a process that does not have specific resonance scales. One consequence is that thermal particles will efficiently damp all waves except those that propagate parallel or perpendicular to the local magnetic field [18]. Equating the rates of damping and cascading yields an angle-dependent wavelength, $\lambda_{\mathrm{c}}$, down to which the fast-mode turbulence can cascade. Using a step function, $\Theta$,the fast-mode turbulence can hence be expected to follow a 3D-spectrum [19]

$$
W\left(k, \Omega_{k}\right)=W_{0} k^{-3.5} \Theta\left(k-\frac{2 \pi}{\lambda_{\max }}\right) \Theta\left(\frac{2 \pi}{\lambda_{\mathrm{c}}}-k\right) .
$$




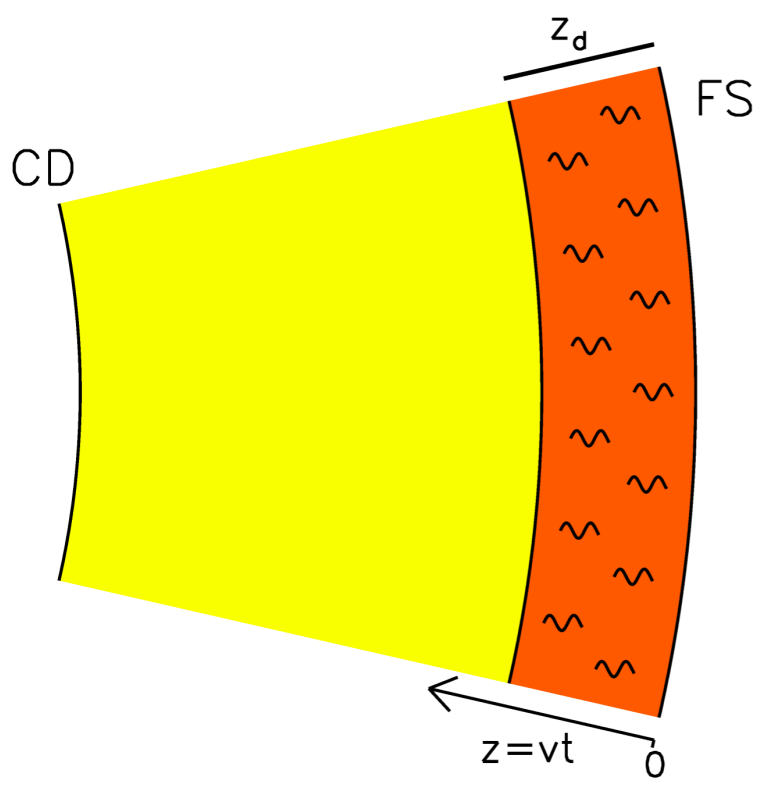

Figure 1: Schematic representation of the scenario. In a region of thickness $z_{d}$ behind the forward shock (FS) turbulence can reaccelerate electrons. Figure taken from [10], reproduced with permission, (c)ESO.

We calculate the momentum diffusion coefficient for an isotropic distribution of electrons as

$$
D_{p}=\int d \mu \frac{p_{\perp}^{2} v_{\perp}^{2}}{8 B_{0}^{2}} \int d k k^{2} \oint d \Omega_{k} k_{\|}^{2} W\left(k, \Omega_{k}\right) R\left(k, \Omega_{k}\right),
$$

where $\perp$ and $\|$ refer to projections perpendicular and parallel to the local mean magnetic field, $B_{0}$, and $\mu=\cos \theta$ reflects the pitch angle relative to it [20]. The resonance function $R\left(k, \Omega_{k}\right)$ includes the effects of orbit perturbations [21]. The corresponding time scale of stochastic acceleration is independent of momentum. It does rely on the isotropy of the particle distribution function, though. Calculating the isotropization time scale is beyond the scope of this paper, but we know that isotropization becomes slower at high particle energies, and therefore our derivation should be realistic only for low-energy particles.

Reaccelerating energetic particles is another damping process for fast-mode turbulence that we have not yet considered. The cosmic-ray induced damping of the waves must be slower than cascading, otherwise the fast-mode cascade would terminate. Using the associated wavelength limit we find as realistic estimate of the acceleration timescale

$$
\tau_{\mathrm{acc}, \mathrm{rev}} \simeq \frac{\lambda_{\max }}{4 \pi c_{\mathrm{s}}} \frac{U_{\mathrm{th}} U_{\mathrm{cr}, \mathrm{acc}}}{U_{\mathrm{fm}}^{2}} \simeq\left(8 \cdot 10^{7} \mathrm{~s}\right)\left(\frac{\lambda_{\max }}{10^{16} \mathrm{~cm}}\right)\left(\frac{c_{\mathrm{s}}}{10^{3} \mathrm{~km} / \mathrm{s}}\right)^{-1} \frac{U_{\mathrm{th}} U_{\mathrm{cr}, \mathrm{acc}}}{10 U_{\mathrm{fm}}^{2}},
$$

where $U_{\text {cr,acc }}$ denotes the energy density in cosmic rays that experience acceleration, $U_{\text {th }}$ is the thermal energy density, $U_{\mathrm{fm}}$ that in fast-mode waves, and $\lambda_{\max }$ is their driving scale. The true acceleration time is thus a few years, and stochastic reacceleration will operate for only a few acceleration times. As the turbulence is driven at the shock and then advects downstream, we must expect that strong fast-mode turbulence exists only in a thin layer of a few $\lambda_{\max }$ in thickness.

\section{Calculation of electron spectra}

The spatial transport of relativistic electrons behind the forward shock of SNR is provided by both advection and diffusion. We concern ourselves with GeV-scale electrons whose spatial- 


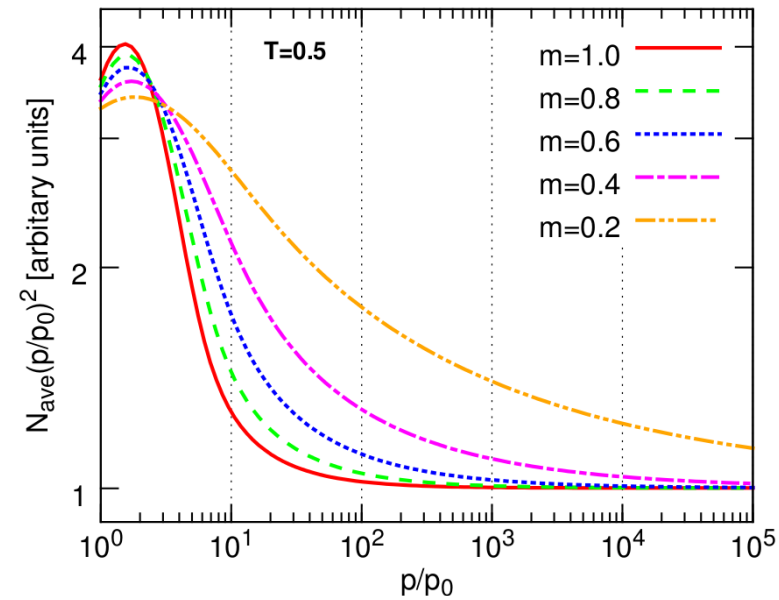

Figure 2: Averaged electron number density, $N_{\text {ave }}$, plotted for various power indices of the momentum diffusion coefficient, $m$, but fixed $T=0.5$. Figure taken from [10], reproduced with permission, (C)ESO.

diffusion coefficient is most likely very small inside SNR. The situation can then be described in the local shock rest frame, i.e. with spatial coordinate $z=r_{\mathrm{sh}}(t)-r$.

Figure 1 summarizes the scenario: In a thin layer of thickness $z_{d}$, determined by cascading and damping of the turbulence behind the forward shock, turbulence subjects electrons to stochastic reacceleration. On account of the dominance of advection over diffusion, shock acceleration at $z=0$ provides accelerated electrons that are fed into the orange-shaded region of re-acceleration and leave it after time $t=z_{d} / v_{\text {adv }}$. Electrons follow an $z$ - $t$ characteristic, and therefore the entire spatial dependence of the electron density is given by the time evolution of the spectrum. The continuity equation then collapses to an initial-value problem of spectral evolution of $N(p, t)$, where

$$
4 \pi r^{2} N(r, p, t)=N(p, t) \delta\left(z-v_{\mathrm{adv}} t\right)
$$

and

$$
\frac{\partial N(p, t)}{\partial t}=\frac{\partial}{\partial p}\left(D_{p}(p) p^{2} \frac{\partial}{\partial p}\left(\frac{N(p, t)}{p^{2}}\right)\right) .
$$

Here, $D_{p}(p)$ is the diffusion coefficient in momentum space. We established that only transit-time damping of fast-mode waves may be fast enough to modify particle spectra inside SNR. Assuming isotropy of the particle distribution function, we found the acceleration time, $\tau_{\mathrm{acc}}$, independent of momentum. Expecting that this assumption breaks down for particles of higher energy, we introduce a dimensionless function, $f(p)$, to set

$$
D_{p}(p)=\frac{p^{2}}{\tau_{\text {acc }}} f(p) \quad \text { with } \quad f(p)= \begin{cases}1 & \text { for } p \leq p_{0} \\ \left(\frac{p}{p_{0}}\right)^{-m} & \text { for } p \geq p_{0}\end{cases}
$$

The form of the momentum diffusion coefficient (Eq. 3.3) permits rewriting the reduced continuity equation (3.2) in dimensionless coordinates,

$$
\frac{\partial N}{\partial x}=\frac{\partial}{\partial \tilde{p}}\left(f(\tilde{p}) \tilde{p}^{4} \frac{\partial}{\partial \tilde{p}} \frac{N}{\tilde{p}^{2}}\right) \quad \text { with } \quad x=\frac{t}{\tau_{\text {acc }}} \quad \text { and } \quad \tilde{p}=\frac{p}{p_{0}} .
$$

Solutions are sought for $0 \leq x \leq T=z_{d} / v_{\text {adv }} / \tau_{\text {acc }}$, where $T$ is the total available time in units of the acceleration time, previously estimated to be at most a few. The spatial variation of the radio 


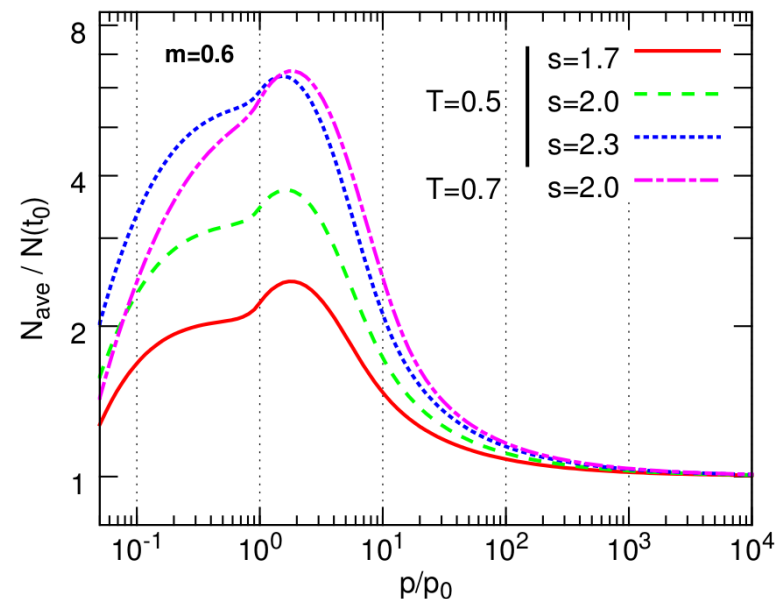

Figure 3: Averaged electron number density, $N_{\text {ave }}$, normalized by the initial distribution, $N\left(t_{0}\right)=N_{0} p^{-s}$, plotted for different initial indices, $s$. Figure taken from [10], reproduced with permission, (c)ESO.

spectrum will probably not be resolvable by current radio observatories. Hence, it may suffice to calculate the average spectrum with the region of strong turbulence (colored orange in Figure 1), for which we need to integrate the particle number density over time:

$$
N_{\text {ave }}(\tilde{p}, T)=\frac{1}{T} \int_{0}^{T} N(\tilde{p}, x) d x .
$$

In the following we set $N_{0}=1$ and initially $N(p, t=0)=N_{0} p^{-2}$. In Figure 2 we present the integrated particle number density, $N_{\text {ave }}$, for various $m$ and fixed time $T=0.5$. To be noted is that a spectral bump appears near $p_{0}$ that has a high-energy tail that extends over more than 2 decades in momentum. To the outside observer that would appear as a concave spectrum.

The question arises to what degree the spectral modifications imposed by stochastic reacceleration depend on the initial spectrum produced at the shock. As test for that we plot in figure 3 the modification factor $N_{\text {ave }} / N(p, t=0)$. For ease of exposition, the initial spectrum is assumed to follow a power law, $N\left(t_{0}\right)=N_{0} p^{-s}$, where we vary the index $s$. The form of the momentum diffusion coefficient is as in Equation 3.3 with fixed $m$. We observe that the choice of initial spectral index determines mainly the amplitude of the spectral bump, whereas its shape is weakly affected. There is some degeneracy between the parameters $m$ and $T$. The initial conditions and the details of diffusive acceleration at the shock are thus largely irrelevant for the spectral characteristics provided by stochastic reacceleration in SNR.

Let us now discuss the radio synchrotron spectrum. In a strongly turbulent magnetic field with $\delta B \gtrsim B_{0}$ the standard synchrotron emissivity is not applicable. We instead use an analytical approximation to the synchrotron emissivity for a turbulent field with Gaussian distribution of amplitudes of scale $B_{\mathrm{rms}}$ and characteristic frequency $v_{c}=v_{0}\left(B_{\mathrm{rms}}\right)$,

$$
P_{\text {eff }} \simeq C B_{\text {rms }} \sqrt{\frac{2}{\pi}}\left(\frac{v}{v_{c}}\right)^{1 / 3} \exp \left(-\frac{3}{2}\left(\frac{v}{v_{c}}\right)^{2 / 3}\right)\left(1+1.65\left(\frac{v}{v_{c}}\right)^{0.42}\right)^{0.53} .
$$

Having established that the choice of initial particle spectrum plays a minor role and can be compensated with adjustments in the dimensionless time, we calculate radio spectra only for $N(p, t=0)=N_{0} p^{-2}$, i.e. the high-frequency spectral index is $\alpha=-0.5$. As we use a dimensionless momentum coordinate, the synchrotron frequency is also dimensionless and normalized to 
the synchrotron frequency $v_{x}$ of electrons of momentum $p_{0}$ in a magnetic field of amplitude $B_{\mathrm{rms}}$,

$$
v_{x}=v_{0}\left(B_{\mathrm{rms}}, p_{0}\right)=\frac{3 e}{4 \pi m_{e}^{3} c^{3}} B_{\mathrm{rms}} p_{0}^{2} .
$$

The radio spectral index of the inner region, shaded yellow in Figure 1, must be calculated with $N(\tilde{p}, x=T)$ and is shown in Figure 4. Note that it is at the same time the radio spectrum at the inner edge of the region of reacceleration, and so it reflects the final state of the electron spectrum after experiencing momentum diffusion for a time $x=T=z_{d} / v_{\text {adv }} / \tau_{a c c}$. Vis-à-vis we present the radio spectral index of the region in which we expect substantial momentum diffusion (shaded red in Figure 1), i.e. computed using the average electron spectrum $N_{\text {ave }}$.
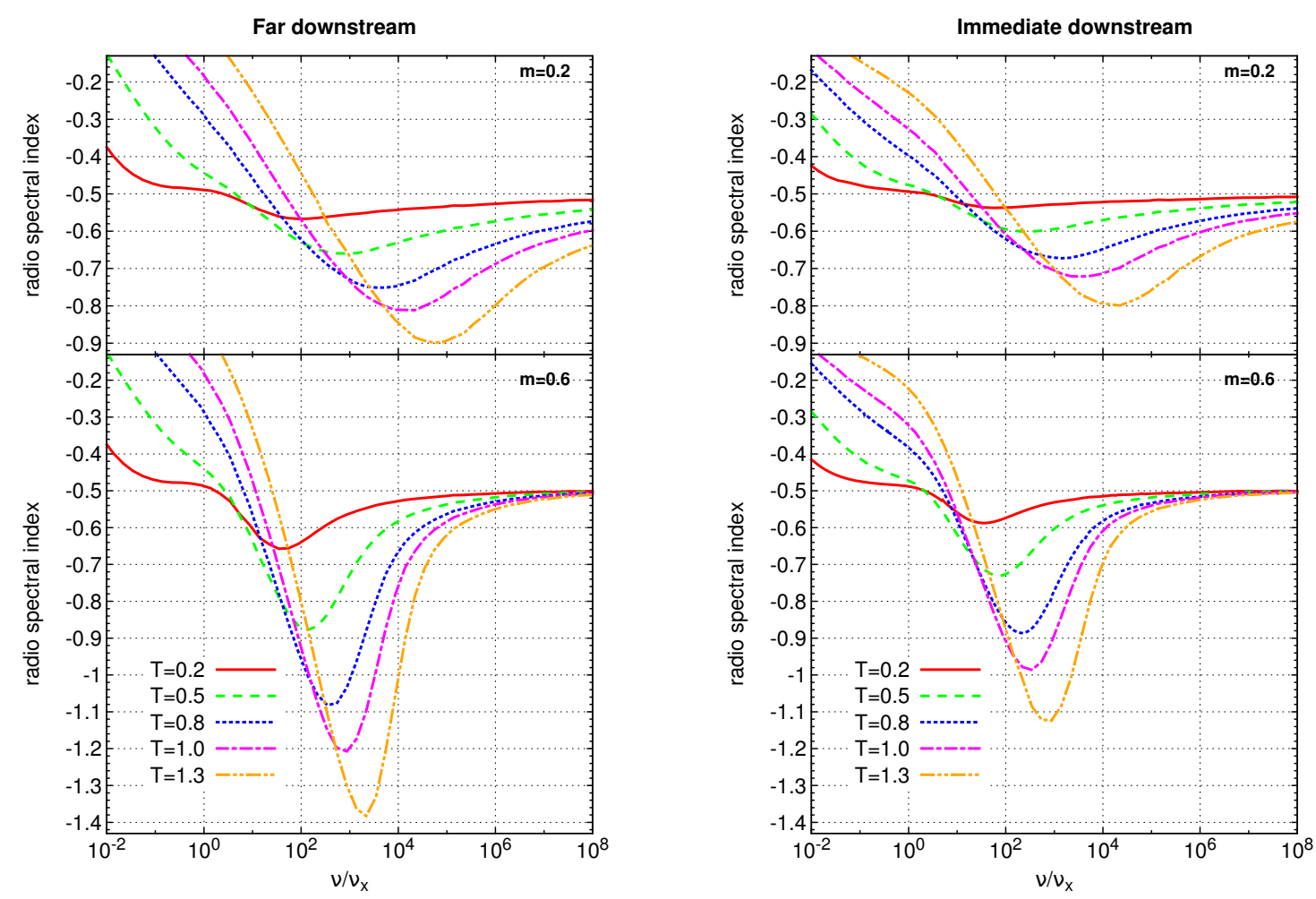

Figure 4: Left panel: Radio spectral index of the far downstream region of an SNR, plotted for different times $T$ and 2 choices of $m$. Right panel: Radio spectral index of the shell where reacceleration occurs, plotted for different times $T$ and 2 choices of $m$. Figures taken from [10], reproduced with permission, (c)ESO.

\section{Summary and conclusions}

We have investigated the role of stochastic reacceleration in SNR with a view to probe whether or not it can account for the wide range of radio spectral indices observed among the more than 200 galactic SNR [3]. The momentum-diffusion coefficient is calculated for 3 types of turbulence, among which only transit-time damping of fast-mode waves operates on timescales of one or a few years. We numerically solved a reduced transport equation and found that cosmic-ray spectra 
develop a bump whose shape is largely independent of the initial spectrum assumed at the forward shock. Finally, synchrotron spectra are derived using an emissivity appropriate for a turbulent magnetic field with Gaussian distribution of amplitudes. Our findings can be summarized as follows:

1) Less than one acceleration time is needed to soften the radio spectrum to $\alpha \simeq-0.65$. As the thickness of the acceleration region is $z_{d}=v_{\mathrm{adv}} T \tau_{\mathrm{acc}}$, for a reacceleration time of a few years and an advection speed of $1000 \mathrm{~km} / \mathrm{s}$ we find that a thickness of $z_{d} \approx 3 \cdot 10^{-3} \mathrm{pc}$ is sufficient which in most cases is not resolvable.

2) If the increase of the reacceleration timescale with momentum is slow, i.e. $m$ is small (cf. Eq. 3.3), soft radio spectra with very little curvature can be maintained over 3 decades in frequency. In contrast, for $m=0.6$ spectral curvature is much stronger and should be detectable, in particular from the far-downstream region.

3) The softest spectra are observed at a few hundred to a thousand $v_{x}$ (cf. Eq. 3.7). For soft radio spectra from SNR it is therefore sufficient, if $v_{x} \approx 10 \mathrm{MHz}$, corresponding to $p_{0} \approx 150 \mathrm{MeV} / c$ for $B_{\mathrm{rms}} \approx 25 \mu \mathrm{G}$.

Acknowledgements: We acknowledge support by the Helmholtz Alliance for Astroparticle Physics HAP funded by the Initiative and Networking Fund of the Helmholtz Association.

\section{References}

[1] Reynolds, S. P. 2008, Ann. Rev. Ast. Astroph., 46, 89

[2] Blandford, R. \& Eichler, D. 1987, Phys. Rep., 154, 1

[3] Green, D. A. 2009, Bulletin of the Astronomical Society of India, 37, 45

[4] Stroman, T., Pohl, M., \& Niemiec, J. 2009, ApJ, 706, 38

[5] Giacalone, J. \& Jokipii, J. R. 2007, ApJ Lett., 663, L41

[6] Inoue, T., Asano, K., \& Ioka, K. 2011, ApJ, 734, 77

[7] Liu, S., Fan, Z.-H., Fryer, C. L., Wang, J.-M., \& Li, H. 2008, ApJ Lett., 683, L163

[8] Bell, A. R. 2004, MNRAS, 353, 550

[9] Mizuno, Y., Pohl, M., Niemiec, J., et al. 2011, ApJ, 726, 62

[10] Pohl, M., Wilhelm, A., \& Telezhinsky, I. 2015, A\&A, 574, A43

[11] Winske, D. \& Leroy, M. M. 1984, J. Geo. Res., 89, 2673

[12] Luo, Q. \& Melrose, D. 2009, MNRAS, 397, 1402

[13] Shalchi, A., Koda, T. Å., Tautz, R. C., \& Schlickeiser, R. 2009, A\&A, 507, 589

[14] Shalchi, A. 2012, Phys. Pl., 19, 102901

[15] Ptuskin, V. S. \& Zirakashvili, V. N. 2003, A\&A, 403, 1

[16] Lynn, J. W., Quataert, E., Chandran, B. D. G., \& Parrish, I. J. 2013, ApJ, 777, 128

[17] Mizuno, Y., Pohl, M., Niemiec, J., et al. 2014, MNRAS, 439, 3490

[18] Quataert, E. 1998, ApJ, 500, 978

[19] Cho, J. \& Lazarian, A. 2002, Phys. Rev. Lett., 88, 245001

[20] Lynn, J. W., Quataert, E., Chandran, B. D. G., \& Parrish, I. J. 2014, ApJ, 791, 71

[21] Yan, H. \& Lazarian, A. 2008, ApJ, 673, 942 